\title{
Evaluation of soluble CD4 serum levels in patients with anogenital warts treated with intralesional tuberculin injection
}

\author{
Original \\ Hesham A. E. Nada', Mohamed M. Elshabrawy', Elsayed A. E. Hassan ${ }^{3}$, Mohamed Azab ${ }^{4}$ \\ Article \\ Departments of ${ }^{I}$ Dermatology, Venereology and Andrology, ${ }^{2}$ Clinical Pathology, ${ }^{4}$ Andrology, \\ Infertility and Sexually Transmitted Diseases, Faculty of Medicine, Suez Canal University, \\ ${ }^{3}$ Department of Dermatology, Ismailia General Hospital, Ismailia, Egypt.
}

\begin{abstract}
Background: Anogenital warts are growths caused by human papilloma virus infection, which causes significant psychosocial morbidity. Intralesional immunotherapy is a promising treatment that is able to stimulate a delayed-type hypersensitivity reaction to different antigens as well as wart tissue. This treatment is suggested to increase CD4 T helper 1 lymphocyte activity to destroy human papilloma virus.

Objective: To measure the level of serum soluble CD4 (sCD4) in patients with anogenital after intralesional tuberculin purified protein derivative (PPD) injection.

Patients and Methods: This study was carried out as a pre-post interventional study on 49 patients with anogenital warts presented to the dermatology clinic and the andrology clinic, Suez Canal University Hospital. Each patient was injected $10 \mathrm{U}(0.2 \mathrm{ml})$ of PPD intralesionally in the largest or mother wart with 2-week interval till complete cure or six injections, whichever was closer. Serum sCD4 was measured before and after injection using sandwich ELISA technique.

Results: Complete clinical wart recovery was obtained in $26(53 \%)$ patients and mostly was after the sixth session $(60.4 \%)$ of injection. Mean level of serum sCD4 was significantly higher after than before PPD injection (18.47 \pm 10.4 vs. $44.48 \pm 20.67 \mathrm{ng} / \mathrm{ml}$, respectively). Serum sCD4 levels were significantly correlated to clinical response rates, as the higher the level of serum $\mathrm{SCD} 4$, the more the wart recovery.

Conclusion: Intralesional PPD injection is a promising effective modality in the treatment of anogenital warts. CD4 cells may have a role in anogenital wart eradication by PPD injection and significantly related to clinical response rates.
\end{abstract}

Key Words: Anogenital warts, immunotherapy, purified protein derivative, soluble CD4.

Received: 09 September 2020, Accepted: 19 December 2020

Corresponding Author: Mohamed Azab, Department of Andrology, Infertility and Sexually Transmitted Diseases, Faculty of Medicine, Suez Canal University, Egypt, Tel.: +201224560152, E-mail: dr.mohamed_azab@yahoo.com.

ISSN: 2090-6048, 2020

\section{INTRODUCTION}

Viral warts are growths or tumors affecting skin and mucous membranes caused by infection with human papilloma virus $(\mathrm{HPV})^{[1]}$. Anogenital wart infection is considered one of the commonest sexually transmitted infections that occur in the genital, perigenital, and perianal regions in sexually active males and females and cause marked stress, anxiety, and interpersonal difficulties ${ }^{[2]}$.

Regulation of an effective immune reaction against different invading pathogens in human body is mainly dependent on CD4+T cells through release of distinctive cytokines after their activation and differentiation on exposure to those pathogens and also through its ability to stimulate different cell types as innate immune cells, cytotoxic T cells, B-lymphocytes and non immune cells ${ }^{[3]}$.

Therapeutic options in anogenital wart management depend on many factors like size, number, distribution and morphology of lesions, as well as treatment adverse effects, cost, availability and convenience, physician experience, and patient needs and preference. The general management of warts includes one of two methods:the first is the ordinary destructive method and the second is immunotherapy ${ }^{[4]}$.

Immunotherapy is a type of treatment that depends on using antigens that stimulate or suppress immune system to help in the treatment of malignancies, infections, immunological disorders, andothers ${ }^{[5]}$. Immunotherapy in anogenital warts uses the capacity of the immune system to recognize certain viral, fungal, and bacterial antigens to initiate a delayed hypersensitivity reaction, which expands the ability of the immune system to perceive and remove the HPV ${ }^{[6]}$.

Cell-mediated immune response had been reported as 
a suggested immune change found in patients with genital wart responding to treatment ${ }^{[7]}$. The CD4+helper $\mathrm{T}$ cell is stimulated through antigen-presenting MHCII molecules, which leads to adaptive humoral immunity effective against warts ${ }^{[8]}$.

Purified protein derivative (PPD) has a role in the treatment of viral warts and being capable of eliciting a delayed-type hypersensitivity at site of injection into patients previously sensitized with tuberculosis bacilli, as previously sensitized CD4+ $\mathrm{T}$ lymphocytes, attracted to the skin test site, proliferate and produce cytokines ${ }^{[9]}$.

Intralesional PPD produces a powerful proinflammatory impulses and appeals antigen-presenting cells, which identify HPV fragments in the affected tissue causing a robust adaptive immune reaction that eradicates the wart infection.PPD has been found to be associated with the production of Thelper 1 cytokines such as interleukin (IL)4 , IL-5, IL-8, IL-12, interferon (IFN)- $\gamma$, and tumor necrosis factor $\alpha$, which stimulate cytotoxic and NK cells that produce a destructive immune reaction against HPV ${ }^{[10]}$.

Rahim et al. ${ }^{[11]}$ assessed CD4 and CD8 levels in serum of patients infected with genital warts and reported that level of CD4 is lower in patients with genital warts thanincontrols in contrast to level of CD8, which was found to be higher in patients than in control group; statistical relationships were significant.

The current study was designed to find out the role of CD4 in the effect of PPD in treatment of anogenital warts through measurement of the level of serum soluble CD4 (sCD4) before and after intralesional injection of tuberculin PPD in anogenital warts.

\section{PATIENTS AND METHODS}

This study was a pre-post interventional study carried out on patients with anogenital warts recruited from the andrology clinic and the dermatology clinic, Suez Canal University Hospital, Faculty of Medicine, Suez Canal University, during the period from February 2019 to March 2020. A total of 49 patients who accepted to participate in this study according to planned eligibility criteria were randomly chosen. Patients included in the study were male patients with diagnosis of external anal or genital warts and aged more than 18 years. Patients excluded from the study were patients with active tuberculosis, chronic or debilitating diseases, andpatients receiving immunosuppressive drugs, systemic corticosteroids, or other therapeutic modalities for anogenital warts, especially immunotherapy during the time of the study or in the last 3 months.

This study was approved by Ethical Committee Board of the Faculty of Medicine, Suez Canal University. Ethical Committee approval number was 3581, dated 9/9/2018. Clear informative medical consent containingaim of the study, rationale, objectives, methodology, expected benefits, and complications of the study was signed by all patients.

All patients were subjected to the following:thorough history taking was obtained and included personal history, present history (duration of infection, sites affected, illegal or extramarital relationships, presence of similar lesions in partners or current affection with other sexually transmitted infections, and previous therapeutic modalities for warts), past medical,and drug history to exclude conditions that interfere with tuberculin PPD injection.

Local examination included carful genital inspection to define number, site, and size of anogenital warts and detection of any genital dermatomes present. Total body skin examination was also performed to exclude presence of other dermatological conditions or any distant warts.

\section{Tuberculin purified protein derivative injection}

The vaccine used wasPPD of a human strain of mycobacterium tuberculosis. It was manufactured by Vacsera Company (Cairo, Egypt). Each vial contained 2-ml solution, and concentration was 5TU (tuberculin unit) per $0.1 \mathrm{ml}$ solution.

Tuberculin test was done first by intradermal injection of 5TU $(0.1 \mathrm{ml}$ solution) of tuberculin PPD in the left forearm. Presence of a well-defined indurated bleb at the site of injection measured about $10 \mathrm{~mm}$ in diameter within $48-72 \mathrm{~h}$ after injection meant that patient was sensitized ${ }^{[12]}$.

After tuberculin test, sensitized patients were injected with tuberculin PPD intralesionally; $10 \mathrm{U}(0.2 \mathrm{ml}$ solution $)$ were used per dose in the largest wartsevery 2 weeks till complete clinical cure or maximum of six injections, whichever was closer ${ }^{[13]}$.

The clinical assessment was performed by the researchers before the study begins and every injection session. Assessment was done for warts in the injected sites and warts in distant anatomical sites.

Assessment of the result of injection was done using the following scale ${ }^{[14]}$ : complete response: complete disappearance of all warts and return of normal skin markings;partial response: regression in warts' size by $25-99 \%$ and no response: regression in size of warts by $0-25 \%$.

The most common adverse effects were pain, edema, fever, mild erythema, and postinflammatory hypopigmentation. These adverse effects happened in a minority of patients and were treated symptomatically by analgesics and anti-inflammatory medications, and in all patients, theseadverse effects didnot lead to discontinuation of the study.

Assessmentof wart recurrence was done by regular follow-up visits every month for 3 months in patients who experienced complete cure of warts to detect any recurrence 
either in same sites of injection or in neighboring sites.

\section{Assessment of serum soluble CD4}

The assay was done for all patients before PPD injection and after complete cure of warts or six sessions of PPD injection whichever was closer, according to the instructions provided by the manufacturer using sCD4 ELISA Kit (Product Id ABIN512865; antibodies-online $\mathrm{GmbH}$, Aachen, Germany).The used sCD4 kithad human reactivity, colorimetric detection using Sandwich ELISA technique. Detection range was $5-100 \mathrm{ng} / \mathrm{ml}$, and the minimum detection limit was $5 \mathrm{ng} / \mathrm{ml}$.

Partial responders and nonresponderswere examined carefully by the researchers and reassessed. Patients were shifted to other management plan such as cryotherapy, ablative $\mathrm{CO} 2$ laser or podophyllin, other immunotherapy materials, or combination of different modalities till complete recovery.

\section{Statistical analysis}

Data were analyzed using IBM SPSS software package, version 20.0. (IBM Corp., Armonk, New York, USA). Qualitative data were described using frequency and percentage. Quantitative data were described using range (minimum and maximum), mean, $\mathrm{SD}$, and median. Significance of the obtained results was judged at the 5\% level.

\section{RESULTS}

This study was carried out on 49 patients with external anogenital warts. The mean age of the studied group was $31.59 \pm 8.34$ years, and most of them were less than 30 years old (55.1\%). Mean duration of warts was 1.0-16.0 months, and $65.3 \%$ of patients had duration less than 8 months. Mean number of warts was $16.76 \pm 11.91$ and ranged from 5 to 34 warts. Warts in distant anatomical sites were found in $18.4 \%$ of patients. Most of the patients completed their sessions and the mean number of sessions was $5.47 \pm 0.53$, and the least number of sessions was three sessions. The most common sites of warts were shaft of penis $(55.2 \%)$ and pubic area $(44.9 \%)$, and the least affected area was perianal area $(6.1 \%)$. Only $20.4 \%$ of patients experienced adverse effects to PPD injection; these adverse effects included local pain, edema, mild erythema, and fever and postinflammatory hypopigmentation (Table 1).

Table 1: Frequency distribution of the studied patients according to sociodemographic characteristics $(\mathrm{N}=49)$

\begin{tabular}{|c|c|}
\hline Parameters & $\mathrm{n}(\%)$ \\
\hline \multicolumn{2}{|l|}{ Age (years) } \\
\hline$\leq 30$ & $27(55.1)$ \\
\hline$>30$ & $22(44.9)$ \\
\hline Minimum-maximum & $21.0-55.0$ \\
\hline Mean \pm SD & $31.59 \pm 8.34$ \\
\hline \multicolumn{2}{|c|}{ Duration of warts (months) } \\
\hline \pm 8 & $32(65.3)$ \\
\hline$>8$ & $17(34.7)$ \\
\hline Minimum-maximum & $1.0-16.0$ \\
\hline Mean \pm SD & $5.73 \pm 4.07$ \\
\hline \multicolumn{2}{|l|}{ Number of warts } \\
\hline Minimum-maximum & $5.0-34.0$ \\
\hline Mean \pm SD & $16.76 \pm 11.91$ \\
\hline \multicolumn{2}{|l|}{ Number of sessions } \\
\hline Minimum-maximum & $3.0-6.0$ \\
\hline Mean \pm SD & $5.47 \pm 1.9$ \\
\hline \multicolumn{2}{|l|}{ Site of anogenital warts } \\
\hline Shaft of penis & $27(55.1)$ \\
\hline Pubic area & $22(44.9)$ \\
\hline Scrotum & $10(20.4)$ \\
\hline Frenulum of penis & $9(18.4)$ \\
\hline Perianal area & $3(6.1)$ \\
\hline \multicolumn{2}{|c|}{ Warts in distant anatomical sites } \\
\hline Present & $9(18.4)$ \\
\hline Absent & $40(81.6)$ \\
\hline
\end{tabular}


Clinical response to intralesional tuberculin PPD injection revealed that $26(53 \%)$ patients completely recovered from warts, 16 (32.7) patients partially recovered, and seven (14.3\%) patients were nonresponders. Complete clinical response occurred in most of the patients at the sixth session of injection (60.4\%).Warts in distant anatomical sites were found in six of the 26 completely recovered patients; five $(83.3 \%)$ of them totally recovered from these distant warts. Intralesional tuberculin PPD injection in warts is a safe method with minimal tolerable adverse effects. In the present study, we found that $79.6 \%$ of patients had no adverse reactions to tuberculin, and adverse effects were found only in $20.4 \%$, in the form of pain $(100 \%)$, mild erythema $(80 \%)$, transient postinflammatory hypopigmentation (30\%), fever (20\%), and transient edema at the site of injection $(20 \%)$. Recurrence occurred in four $(15.4 \%)$ of the 26 completely recovered patients during the 3-month period after completion of sessions(Table 2).

Table 2: Frequency distribution of the studied patients according to clinical response to purified protein derivative injection, adverse effects and recurrence rate

\begin{tabular}{|c|c|}
\hline Clinical response to PPD & $\mathrm{N}=49[\mathrm{n}(\%)]$ \\
\hline No response $(0-25 \%)$ & $7(14.3)$ \\
\hline Partial response (25-99\%) & $16(32.7)$ \\
\hline Complete response $(100 \%)$ & $26(53)$ \\
\hline \multicolumn{2}{|c|}{ Session of complete response $(\mathrm{N}=26)$} \\
\hline Third session & $1(3.8)$ \\
\hline Fourth session & $3(11.5)$ \\
\hline Fifth session & $6(23.1)$ \\
\hline Sixth session & $16(61.6)$ \\
\hline \multicolumn{2}{|c|}{ Response of distant warts in completely recovered patients $(\mathrm{N}=6)$} \\
\hline Complete response & $5(83.3)$ \\
\hline Partial or no response & $1(16.7)$ \\
\hline \multicolumn{2}{|c|}{ Occurrence of Adverse reactions to PPD injection ( $N=49)$} \\
\hline No adverse reaction & $39(79.6)$ \\
\hline Adverse reaction & $10(20.4)$ \\
\hline \multicolumn{2}{|c|}{ Adverse reactions to PPD injection $(\mathrm{N}=10)$} \\
\hline Pain & $10(100)$ \\
\hline Mild erythema & $8(80)$ \\
\hline Hypopigmentation & $3(30)$ \\
\hline Fever & $2(20)$ \\
\hline Transient edema & $2(20)$ \\
\hline \multicolumn{2}{|c|}{ Recurrence after completeresponse $(\mathrm{N}=26)$} \\
\hline No & $22(84.6)$ \\
\hline Yes & $4(15.4)$ \\
\hline
\end{tabular}

PPD, purified protein derivative.

Level of serum SCD4 was significantly increased after intralesional tuberculin PPD injection as mean serum sCD4 was $18.47 \pm 10.4$ versus $44.48 \pm 20.67 \mathrm{ng} / \mathrm{ml}$ before and after injection, respectively $(P<0.001)$ (Table 3$)$.

Correlation between different demographic parameters and clinical response of intralesional tuberculin PPD injection revealed that age was significantly related to response; the younger the age, the better the response. The number of warts was also significantly related to response, as the smaller the number, the better the response. Duration of warts was also significantly related to response, as the shorter the duration, the better the response $(P<0.001)$. Site of warts was not a determinant factor in clinical response as there was no significant statistical relationship between site and clinical response (Table 4).

Correlation between level of serum sCD4 and clinical 
response revealed that before PPD injection level of sCD4 was not significantly related to clinical response,whereas after injection, serum sCD4 levels were significantly correlated $(P<0.001)$ to response, as the more the clinical response rate, the higher the level of serum sCD4 (Table 5).

Table 3: Comparison of serum levels of serum soluble CD4 before and after injection with intralesional tuberculin purified protein derivative injection $(\mathrm{N}=49)$

\begin{tabular}{|c|c|c|c|c|}
\hline sCD4 (ng/ml) & Before injection $(\mathrm{N}=49)$ & After injection $(\mathrm{N}=49)$ & $t$ & $P$ \\
\hline Minimum-maximum & $7.20-55.42$ & $10.60-82.51$ & & \\
\hline Mean \pm SD & $18.47 \pm 10.4$ & $39.48 \pm 20.67$ & $5.386^{*}$ & $<0.001^{*}$ \\
\hline Median & 19.0 & 29.0 & & \\
\hline Change & \multicolumn{4}{|c|}{$\uparrow 19.01 \pm 35.81$} \\
\hline
\end{tabular}

t,paired ttest.

sCD4, serum soluble CD4.

$P: P$ value for comparing between sCD4 levels before and after injection.

*Statistically significant at $P$ value less than or equal to 0.05 .

Table 4: Comparison of age, number, duration and site of anogenital warts, and serum levels of serum soluble CD4 clinical response after intralesional tuberculin purified protein derivative injection $(\mathrm{N}=49)$

\begin{tabular}{|c|c|c|c|c|c|c|}
\hline \multirow[b]{2}{*}{ Variables } & \multicolumn{6}{|c|}{ Clinical response } \\
\hline & No response $(\mathrm{N}=7)$ & $\begin{array}{l}\text { Partial response } \\
\qquad(\mathrm{N}=16)\end{array}$ & \multicolumn{2}{|c|}{$\begin{array}{c}\text { Complete response } \\
\qquad(\mathrm{N}=26)\end{array}$} & $\begin{array}{l}\text { Test of } \\
\text { significance }\end{array}$ & $P$ \\
\hline Number of warts & & & & & $H$ & $P$ \\
\hline Mean \pm SD & $26.71 \pm 1.98$ & $19.56 \pm 6.87$ & \multicolumn{2}{|c|}{$13.69 \pm 3.66$} & $34.744 *$ & $0.021 *$ \\
\hline Duration of warts (months) & & & & & $H$ & $P$ \\
\hline Mean \pm SD & $12.57 \pm 1.99$ & $11.31 \pm 1.08$ & \multicolumn{2}{|c|}{$4.23 \pm 1.63$} & $37.266^{*}$ & $<0.001^{*}$ \\
\hline Site of warts [n (\%)] & & & & & $\chi^{2}$ & мср \\
\hline Shaft of penis & 57.1 & 43.8 & 16 & 61.5 & 1.336 & 0.524 \\
\hline Pubic area & 57.1 & 56.3 & 9 & 34.6 & 2.397 & 0.295 \\
\hline Scrotum & 0.0 & 37.5 & 4 & 15.4 & 4.332 & 0.078 \\
\hline Frenulum & 0.0 & 18.8 & 6 & 23.1 & 1.572 & 0.520 \\
\hline Perianal & 0.0 & 12.5 & 1 & 3.8 & 1.504 & 0.715 \\
\hline \multicolumn{7}{|l|}{ Serum soluble CD4 (ng/ml) } \\
\hline After PPD injection & & & & & $F$ & $P$ \\
\hline Minimum-maximum & $7.20-40.45$ & $16.45-54.20$ & 15.89 & 5.42 & 2.871 & 0.067 \\
\hline Mean \pm SD & $18.86 \pm 11.82$ & $18.34 \pm 9.22$ & $16.11 \pm$ & 1.88 & & \\
\hline After PPD injection & & & & & $F$ & $P$ \\
\hline Minimum-maximum & $10.60-48.50$ & $15.60-80.64$ & 21.00 & 2.51 & $32.758 *$ & $<0.001 *$ \\
\hline Mean \pm SD & $27.73 \pm 8.82$ & $32.84 \pm 11.46$ & $49.23 \pm$ & 8.37 & & \\
\hline
\end{tabular}

$\chi 2, \chi 2$ test; F, analysis of variance test; H,Kruskal-Wallis test; MC, Monte Carlo; PPD, purified protein derivative; sCD4, serum soluble CD4. $\mathrm{P}$ : $\mathrm{P}$ value for association between clinical response and age, number, duration, site of warts and serum soluble CD4.

*Statistically significant at $P$ value less than or equal to 0.05 . 
Table 5: Comparison of serum levels of serum soluble CD4 and clinical response after intralesional tuberculin purified protein derivative injection $(\mathrm{N}=49)$

\begin{tabular}{|c|c|c|c|c|c|}
\hline \multirow{2}{*}{ sCD4 (ng/ml) } & \multicolumn{5}{|c|}{ Clinical response } \\
\hline & No response $(\mathrm{N}=7)$ & $\begin{array}{l}\text { Partial response } \\
\qquad(\mathrm{N}=16)\end{array}$ & $\begin{array}{l}\text { Complete response } \\
\qquad(\mathrm{N}=26)\end{array}$ & $\begin{array}{c}\text { Test of } \\
\text { significance }\end{array}$ & $P$ \\
\hline Before PPD injection & & & & $F$ & $P$ \\
\hline Minimum-maximum & $7.20-40.45$ & $16.45-54.20$ & $15.89-55.42$ & 2.871 & 0.067 \\
\hline Mean \pm SD & $18.86 \pm 11.82$ & $18.34 \pm 9.22$ & $16.11 \pm 11.88$ & & \\
\hline After PPD injection & & & & $F$ & $P$ \\
\hline Minimum-maximum & $10.60-48.50$ & $15.60-80.64$ & $21.00-82.51$ & $32.758 *$ & $<0.001 *$ \\
\hline Mean \pm SD & $27.73 \pm 8.82$ & $32.84 \pm 11.46$ & $49.23 \pm 18.37$ & & \\
\hline
\end{tabular}

$F$, analysis of variance test; PPD, purified protein derivative; sCD4: serum soluble CD4.

$P$ : $P$ value for association between clinical response and CD4.

* Statistically significant at $P$ value less than or equal to 0.05 .

\section{DISCUSSION}

Anogenital warts are a very common sexually transmitted condition in both males and females that cause marked quality of life deterioration especially sexual life and couple interpersonal communications. AGW are caused by HPV infection, which has more than 100 subtypes; the most common strains are 6 and 11 subtypes ${ }^{[15]}$. Immunotherapy is a promising new line of management of numerous and difficult-to-treat anogenital warts as it can cause complete wart removal with minimal invasion, injury, pain, scarring, and relatively short duration in some cases as well as they improve the host immune reactivity response against HPV, which leads to high recovery and little recurrence rates ${ }^{[13]}$. Immunotherapy is considered an economical therapeutic modality for extensive neglected wart infections and subsequently can be of extraordinary incentive in developing countries ${ }^{[16]}$

In the current study, we used immunotherapy with intralesional tuberculin PPD injection as a method of treatment of anogenital warts. Results revealed that complete removal of warts occurred in the majority of cases (53\%), followed by $32.7 \%$ who had partial response, then $14.3 \%$ had minimal response after about six sessions of injection. These results are in concordance with Mohamed et al..$^{[10]}$, who reported that complete clearanceof warts was observed in 43 (69.4\%) patients, partial response (25-99\%) was observed in $13(20.9 \%)$ patients, and no response $(0-24 \%)$ was observed in six $(9.7 \%)$ patients, whereas Rajashekar et al. ${ }^{[16]}$ found that complete clearance occurred in $35.3 \%$ of patients either on the injection site as well as anatomically distant sites, suggesting that response to PPD injection is a systemic response and not only restricted to the site of the injection.Singh et al. ${ }^{[7]}$ also reported that of
40 patients, $32(80 \%)$ patients showed complete recovery, six $(15 \%)$ patients showed partial recovery, and two (5\%) patients showed no response.

In the same line, Shaheen et al. ${ }^{[17]}$ reported that PPD immunotherapy injection showed $60 \%$ clearance of target warts and distant warts with significant increase in circulating IL4.Nimbalkar et al. ${ }^{[13]}$ was in agreement with our study, asthey found that of 45 patients, 28 $(62.2 \%)$ showed complete clearance, eight (17.8\%) patients showed partial clearance, and nine $(20 \%)$ patients showed no improvement. Higher results were reported by Elela et al. ${ }^{[18]}$, who found that complete clearance was seen in $94.1 \%$ of the cases when intralesional PPD was administered in the wart tissue versus success rates of $96 \%$ with the intradermal injection.

On the contrary, Kus et al. ${ }^{[19]}$ used intralesional tuberculin PPD injection in 18 patients with recalcitrant warts and found that complete cure was found in $5 / 18$ (29\%) patients, partial response was found in 10/18 (59\%) patients, and no response was found in two (12\%) patients. This cure rate is lower than the cure rate in our study and previously mentioned studies, and this can be explained by a small sample size, lower number of sessions, and wider interval of injections ( 3 vs. 2 weeks) in our study.

Cellular immunity has the main responsibility in fighting wart infection. Spontaneously recovering warts show exceptional epidermal and dermal release of CD4+ activated memory lymphocytes in comparison with nonrecovered warts. HPV proteins antibodies have been significantly found in the serum of HPV-infected patients, but the role of these antibodies is uncertain as they do not correlate with the wart regression ${ }^{[20]}$. Additionally, Critchlow et al. ${ }^{[21]}$ found that defective T-cell-mediated 
immune response can be the cause of prolonged and resistant infection with anogenital warts.

In 2017, Singh et al. ${ }^{[7]}$ studied the functional characterizationof HPV6 and HPV11 antigen-specific CD4+and CD8+T-cell responses from serum of patients with anogenital warts and reported that frequency of both CD4+ IFN- $\gamma+$ and CD8+ IFN- $\gamma+$ cellsin patients with anogenital wart was significantly lower than healthy controls.In this study, they recommended that their findings may be helpful in designing future immunomodulation methods that can be used as adjunct immunotherapy to stimulate host immune response in patients of anogenital warts. This might help in prevention of recurrence of warts after treatment ${ }^{[7]}$

In the current work, we measured the level of sCD4 in serum of patient with anogenital warts before and after intralesional tuberculin PPD injection. The levelof serum sCD4 was significantly increased after than before injection as mean serum sCD4 level was $18.47 \pm 10.4$ versus $44.48 \pm 20.67$,respectively $(P<0.001)$. Before tuberculin PPD injection, level of sCD4 was not significantly correlated to clinical cure rates, whereas after injection, serum sCD4 levels were significantly related $(P<0.001)$ to clinical recovery rates, as the more clinical response rate, the higher the level of serum sCD4.

Rahim et $a l^{[11]}$ studied the relationship between serum levels of both sCD4 and sCD8 and genital wart activity, and they reported that level of sCD4 was lower in patients versus controls $(5.47 \pm 0.12$ vs. $10.98 \pm 0.19 \mathrm{ng} / \mathrm{ml}, \quad$ respectively; $\quad P=0.001)$, which was opposite to the level of serum sCD8, which was higher in patients thancontrol group $(38.56 \pm 1.90$ vs. $16.86 \pm 0.23 \mathrm{ng} / \mathrm{ml}$, respectively).

Findings in our study were in concordance indirectly with results reported by Rahim et al. ${ }^{[1]}$ and Singh et $a{ }^{\left[{ }^{[7]}\right.}$, with some difference in study design and management. In the current study, we were the first to measure the level of CD4 cells in patients with anogenital warts in relation to immunotherapy modality. We assumed that CD4 cells had an effective role in mechanism of action of intralesional tuberculin PPD as a treatment method for anogenital warts. This was proved by the higher levels of serum sCD4 before injection than after injection and significant statistical correlation between sCD4 level and rates of clinical response. Another clue for this hypothesis is regression of distant or neighboring warts in other sites rather than injection sites, which denotes that tuberculin PPD intralesional injection causes significant systemic immune reaction may be dependent on CD4 cells and causes wart recovery.

Limitations of the study were the small sample size, making the results difficult to be generalized; sensitivity of the vaccine, as well as ease to be spoiled by heat and light, with continuous need to strict cold chain maintenance; bad patient compliance as intolerance to adverse effects and boring from repeated injections; shame and stigmata of genital wart infections; difficulties in assessment of partners; and data regarding effect of immunotherapy with PPD and level of sCD4 being limited to be compared with our results.

\section{CONCLUSION}

Immunotherapy with intralesional tuberculin PPD is an effective, dependent economic modality of treatment of genital warts with good cosmetic results and lower recurrence rates. Serum sCD4 levels were higher in patients with anogenital warts treated with intralesional tuberculin PPD injection than pretreatment levels and were significantly correlated to wart clinical response rates.

\section{CONFLICT OF INTEREST}

1. PretetJL, CharlotJF, MouginC. Virological and carcinogenic aspects of HPV. Bull Acad Natl Med2007; 191:611-623.

2. LopaschukCC. New approach to managing genital warts. Can Fam Physician 2013; 59:731-736.

3. MahnkeYD, BeddallMH, RoedererM. OMIP-017: human CD4+ helper T-cell subsets including follicular helper cells. Cytometry A2013; 83:439-440.

4. Vender, R., Bourcier, M., Bhatia, N., and Lynde, C. Therapeutic options for external genital warts. J Cutan Med Surg2013; 17 (Suppl 2):S61-S67.

5. Guo L, Zhang $\mathrm{H}$, ChenB. Nivolumab as programmed death-1 (PD-1) inhibitor for targeted immunotherapy in tumor. J Cancer2017; 8:410.

6. Chauhan, P. S., Mahajan, V. K., Mehta, K. S., Rawat, R., and Sharma, V. The efficacy and safety of intralesional immunotherapy with measles, mumps, rubella virus vaccine for the treatment of common warts in adults. Indian Dermatol Online J2019; 10:19.

7. Singh, M., Thakral, D., Kar, H.K., Rishi, N., Sharma, P.K. and Mitra, D.K., Distinct clinicoimmunological profile of patients infected with human papilloma virus genotypes 6 and 11 . Virusdisease 2017. 28:200-204.

8. CrottyS. T follicular helper cell differentiation, function, and roles in disease. Immunity 2014; 41:529-542.

9. MacdonaldG. Harrison's internal medicine, -by AS Fauci, DL Kasper, DL Longo, E. Braunwald, SL Hauser, JL Jameson and J. Loscalzo. Intern Med J2008; 38:932-932. 
10. Mohamed EM, El TaiebMA, Abd El-sabourGA. Intralesional vitamin D3 versus purified protein derivatives in the treatment of multiple cutaneous warts: comparative study. Egypt J Hosp Med2019; 76:3589-3594.

11. RahimS, IbraheamIA, RadiNK. Evaluation of CD4 and CD8 in patients infected with genital wart caused by human papilloma virus in Babylon province/Iraq. J Glob Pharma Technol2017; 9:372-375.

12. NayakS, AcharjyaB. Mantoux test and its interpretation. Indian Dermatol Online J2012; 3:2.

13. Nimbalkar, A., Pande, S., Sharma, R. and Borkar, M., Tuberculin purified protein derivative immunotherapy in the treatment of viral warts. Indian J Drugs Dermatol2016; 2:19.

14. Jaisinghani, A.K., Dey, V.K., Suresh, M.S. and Saxena, A., Bacillus Calmette-Guerin immunotherapy for recurrent multiple warts: an open-label uncontrolled study. Indian $\mathbf{J}$ Dermatol2019; 64:164.

15. LynchMD, CliffeJ, Morris-JonesR. Management of cutaneous viral warts. BMJ2014; 348:g3339.

16. RajashekarTS, AmulyaR., SathishS, KumarS.
Comparative study of intralesional BCG and PPD in the treatment of multiple cutaneous warts. Indian J Clin Exp Dermatol2018; 2018:1-6.

17. Shaheen, M.A., Salem, S.A.M., Fouad, D.A. and El-Fatah, A.A.A. Intralesional tuberculin (PPD) versus measles, mumps, rubella (MMR) vaccine in treatment of multiple warts: a comparative clinical and immunological study. Dermatol Ther2015; 28:194-200.

18. Elela I, ElshahidAR, MosbehAS. Intradermal vs intralesional purified protein derivatives in treatment of warts. Golf J Deramatol Venereol2011; $18: 21-26$.

19. Kus, S., Ergun, T., Gun, D. and Akin, O., Intralesional tuberculin for treatment of refractory warts. J Eur Acad Dermatol2005; 19:515-516.

20. MajewskiS, JablonskaS. Immunology of HPV infection and HPV-associated tumors. Int $\mathrm{J}$ Dermatol1998; 37:81-95.

21. Critchlow, C.W., Hawes, S.E., Kuypers, J.M., Goldbaum, G.M., Holmes, K.K., Surawicz, C.M. and Kiviat, N.B., Effect of HIV infection on the natural history of anal human papillomavirus infection. AIDS1998; 12:1177-1184. 